\title{
Revisiting the Analysis of the Isochronous Mass Measure- ments of Uranium Fission Fragments at the ESR
}

\author{
R. S. Sidhu ${ }^{1,2, *}$, R. J. Chen ${ }^{1,3}, Y u$. A. Litvinov ${ }^{1}, Y . H$. Zhang $^{3}$, and the FRS-ESR Collaboration \\ ${ }^{1}$ GSI Helmholtzzentrum für Schwerionneforschung, 64291 Darmstadt, Germany \\ ${ }^{2}$ Physikalisches Institut, Ruprecht-Karls-Universität, 69120 Heidelberg, Germany \\ ${ }^{3}$ Key Laboratory of High Precision Nuclear Spectroscopy and Center for Nuclear Matter Science, In- \\ stitute of Modern Physics, Chinese Academy of Sciences, Lanzhou 730000, P. R. China
}

\begin{abstract}
The re-analysis of experimental data on mass measurements of uranium fission products obtained at the ESR in 2002 is discussed. State-of-the-art data analysis procedures developed for such measurements are employed.
\end{abstract}

\section{Introduction}

Nuclear mass is a fundamental property of an atomic nucleus. Precise measurement of nuclear masses is crucial for better understanding nuclear structure and for modelling nucleosynthesis processes in the cosmos [1]. Storage ring mass spectrometry is one of the techniques for direct mass measurements [2]. In addition to masses, also the halflives of exotic nuclei can simultaneously be measured [3]. The central formula for the storage ring mass spectrometry (first order approximation) is [4]:

$$
\frac{\Delta T}{T}=\frac{1}{\gamma_{t}^{2}} \frac{\Delta m / q}{m / q}-\left(1-\frac{\gamma^{2}}{\gamma_{t}^{2}}\right) \frac{\Delta v}{v}
$$

where $T, m / q, v, \gamma_{t}$, and $\gamma$ are revolution time, mass-to-charge ratio, velocity, transition energy, and the relativistic Lorentz factor, respectively.

By minimising the second term on the right side of equation (1), the mass-to-charge ratio can be determined. In the case of Isochronous Mass Spectrometry (IMS) [5], the ions of interest are injected with velocities corresponding to $\gamma=\gamma_{t}$, where the "transition energy" $\gamma_{t}$ is the characteristic quantity of the heavy-ion storage ring. Then, the revolution time (or revolution frequency) of such ions becomes independent of their velocity spread, $\Delta v / v$. The revolution times can be measured either with a dedicated secondary-electron-based timeof-flight (ToF) detector [6, 7] or non-destructively with the help of fast Schottky detector $[8,9]$. The masses of unknown nuclides are determined by comparing their revolution times to those of the nuclei with precisely known masses. In this way, storage rings can be used for the measurements on exotic nuclei with short half-lives and tiny production rates. The IMS measurements were pioneered at the ESR [10,11]. Due to the construction of the new FAIR facility, there were no IMS measurements in the ESR for about ten years. However, in the last decade, a series of precision measurements has been conducted at the CSRe in Lanzhou [12-15]. Dedicated methods were developed there to analyse the experimental data [16-18].

\footnotetext{
*e-mail: R.S.Sidhu@gsi.de
} 
In this work, we report on the attempt to employ this state-of-the-art analysis to the IMS data obtained at the ESR.

\section{Experiment and data analysis}

The experiment was performed at GSI in 2002 [19]. Relativistic ${ }^{238} \mathrm{U}$ beam with an average intensity of $10^{9}$ particles per spill impinged a $1032 \mathrm{mg} / \mathrm{cm}^{2}$ Be target placed at the entrance of the Fragment Separator, FRS [20]. Secondary ions were produced via abrasion-fission of the primary beam. Neutron-rich nuclei of interest were separated in the FRS, transported to and injected into the ESR [21]. All produced nuclei, the "cocktail beam", lying within the injection acceptance were stored in the ESR. The ESR was tuned into the isochronous mode with $\gamma_{t} \sim 1.43$. Three settings centred on ${ }^{130,133,135} \mathrm{Sn}$ ions have been employed. The details of typical FRS-ESR experiments can be found in [22-24].

The revolution times of the circulating ions were determined with a ToF detector [6], consisting of a thin carbon foil combined with two microchannel plates (MCP). The secondary electrons released from the carbon foil due to the passage of the ions were creating timing signals on the MCPs. The data acquisition was triggered by the injection of the ions into the ESR. The output of the ToF detector, including the timing signals, was digitised with $20 \mathrm{GS} / \mathrm{s}$ for the entire measurement period of $1 \mathrm{~ms}$. We re-analysed all data. A part of revolution time spectrum for the ${ }^{135} \mathrm{Sn}$ setting is shown in Figure 1(a). The particle identification was done by comparing with a simulated spectrum.
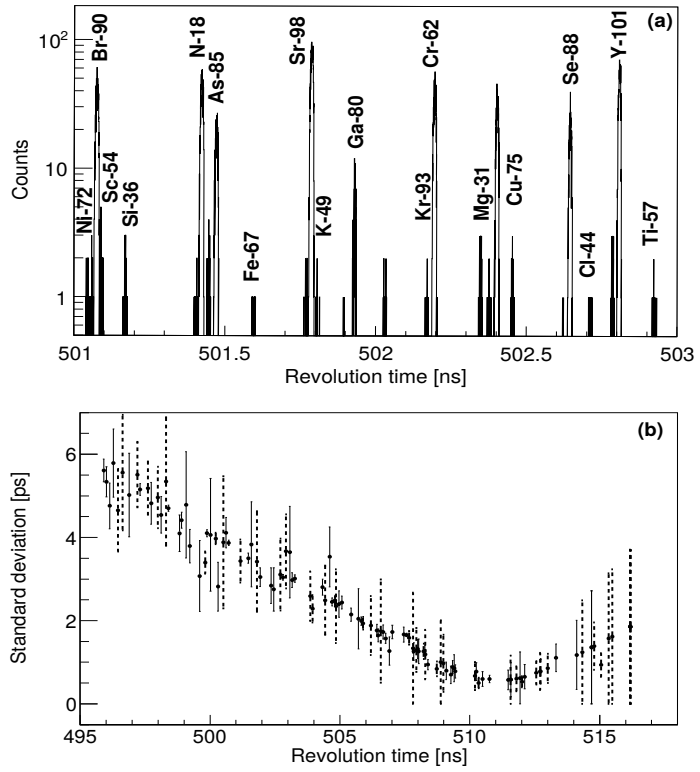

Figure 1. (a) The revolution time spectrum of ${ }^{238} \mathrm{U}$ fission fragments zoomed in at $501 \mathrm{~ns} \leq \mathrm{t} \leq 503 \mathrm{~ns}$. (b) Standard deviations of the revolution time peaks for the full spectrum

A mass resolving power of about 178000 has been achieved without applying any further second order corrections. This is sufficient to directly separate isobars and even low-lying isomers. Figure 1(b) shows the standard deviations of the revolution time peaks for the full spectrum with a clear minimum of $\sim 0.5 \mathrm{ps}$. As a result, several previously unknown masses of exotic nuclei could be determined with high precision. The data analysis is in progress. 


\section{Outlook}

Storage rings have proven to be an efficient tool for precision measurements of masses and lifetimes of exotic nuclei. Currently there are three storage ring facilities in the world, the ESR at GSI, the CSRe at IMP and the R3-storage ring at RIKEN Nishina Center [2]. A variety of exciting physics cases with Highly Charged Ions (HCIs) are being pursued [25-28]. The high discovery potential of such studies motivated the construction of next-generation facilities like FAIR in Germany [29] and HIAF in China [30]. Storage ring mass and lifetime spectroscopy is in the focus at all present and future facilities [31]. In this context, the present work is important to identify whether tools and techniques developed at different setups can efficiently and commonly be used.

This work is in part supported by the European Research Council (ERC) under the EU Horizon 2020 research and innovation programme (ERC CG 682841 “ASTRUm”).

\section{References}

[1] K. Blaum, Phys. Rep 425, 1 (2006)

[2] Y. H. Zhang et al., Phys. Scripta 91, 073002 (2016)

[3] F. Bosch et al., Prog. Part. Nucl. Phys. 73, 84 (2013)

[4] B. Franzke et al., Mass Spectrometry Reviews 27, 428 (2008)

[5] H. Wollnik, Nucl. Instr. Meth. B 26, 267 (1987)

[6] J. Trötscher et al., Nucl. Instr. Meth. B 70, 455 (1992)

[7] B. Mei et al., Nucl. Instr. Meth. A 624, 109 (2010)

[8] F. Nolden et al., Nucl. Instr. Meth. A 659, 69 (2011)

[9] X. C. Chen et al., Nucl. Instr. Meth. A 826, 39 (2016)

[10] M. Hausmann et al., Nucl. Instr. Meth. A 446, 569 (2000)

[11] M. Hausmann et al., Hyperfine Interactions 132, 289 (2001)

[12] Y. M. Xing et al., Phys. Lett. B 781, 358 (2018)

[13] C. Y. Fu et al., Phys. Rev. C 98, 014315 (2018)

[14] Y. H. Zhang et al., Phys. Rev. C 98, 014319 (2018)

[15] X. Xu et al., Phys. Rev. C 99, 064303 (2019)

[16] X. L. Tu et al., Nucl. Instr. Meth. A 654, 213 (2011)

[17] P. Shuai et al., Nucl. Instr. Meth. B 376311 (2016)

[18] Y. M. Xing et al., Nucl. Instr. Meth. A 941, 162331 (2019)

[19] M. Matos, PhD Thesis, University of Giessen (2004)

[20] H. Geissel et al., Nucl. Instr. Meth. B 70, 286 (1992)

[21] B. Franzke, Nucl. Instr. Meth. B 24/25, 18 (1987)

[22] H. Geissel et al., Eur. Phys. J. Special Topics 150, 109 (2007)

[23] B. H. Sun et al., Phys. Lett. B 688, 294 (2010)

[24] R. Knöbel et al., Eur. Phys. J. A 52, 138 (2016)

[25] T. Stöhlker et al., Nucl. Instr. Meth. B 365, 680 (2015)

[26] Y. A. Litvinov et al., Nucl. Instr. Meth. B 317, 603 (2013)

[27] M. Lestinsky et al., Eur. Phys. J. Special Topics 225, 797 (2016)

[28] R. Singh Sidhu et al., EPJ Web of Conf. 178, 01003 (2018)

[29] M. Durante et al., Phys. Scripta 94, 033001 (2019)

[30] J. C. Yang et al., Nucl. Instr. Meth. B 317, 263 (2013)

[31] P. M. Walker et al., Int. J. Mass Spectrom. 349-350, 247 (2013) 